\title{
Secondary hemorrhage after different modes of hysterectomy
}

\author{
P. G. Paul • Anil Sakhare Panditrao - Shabnam Khan • \\ Prathap Talwar • Harneet Kaur - Sheetal Barsagade
}

Received: 5 March 2013 /Accepted: 26 August 2013 /Published online: 10 September 2013

(C) Springer-Verlag Berlin Heidelberg 2013

\begin{abstract}
Secondary hemorrhage after hysterectomy is rare but a life-threatening complication. The aim of this study is to estimate the cumulative incidence, patient characteristics, and potential risk factors of secondary hemorrhage after abdominal, vaginal, and laparoscopic hysterectomies. We did a retrospective observational study in which 1,623 cases of total laparoscopic hysterectomy (TLH), 963 cases of total abdominal hysterectomy (TAH), and 1,171 cases of vaginal hysterectomy (VH) were analyzed. Of the total 37 hemorrhages following hysterectomies, 23 were after TLH, 8 following $\mathrm{VH}$, and 6 were after TAH. The cumulative incidence of secondary hemorrhage after any type of total hysterectomies was $0.98 \%$. TLH was associated with the highest risk of secondary hemorrhage $(1.51 \%)$ followed by VH $(0.68 \%)$ and TAH group $(0.62 \%)$. The relative risk of secondary hemorrhage following TLH compared to TAH and VH were 2.3 and 2.1, respectively. Both were statistically significant. The average size of the uterus in the TLH group was $516.7 \mathrm{~g}$, and in the TAH and VH group, it was 140 and $142.5 \mathrm{~g}$, respectively, which was statistically significant. The median time interval between hysterectomy and secondary hemorrhage was 11 days in TAH and $\mathrm{VH}$ group and 13 days in TLH group. Our data suggest that secondary hemorrhage is rare but may occur more often after TLH than after other hysterectomy approaches. Whether it is related to the application of thermal energy to tissues which cause more tissue necrosis and devascularization than sharp colpotomies in the TAH and $\mathrm{VH}$ groups is unclear. Large size of uteri, excessive use of energy source for uterine artery, and colpotomy may play a role.
\end{abstract}

P. G. Paul $(\bowtie) \cdot$ S. Khan $\cdot$ P. Talwar $\cdot$ H. Kaur $\cdot$ S. Barsagade

Paul's Hospital, Kochi, Kerala, India

e-mail: drpaulpg@gmail.com

A. Sakhare Panditrao

Dr.Shankar Rao Chavan, Govt Medical College,

Nanded, Maharashtra, India
Keywords Laparoscopic hysterectomy $\cdot$ Abdominal hysterectomy $\cdot$ Vaginal hysterectomy $\cdot$ Secondary hemorrhage

\section{Background}

Secondary hemorrhage after hysterectomy is rare but a lifethreatening complication which may require prompt medical and surgical intervention. Although the overall incidence of secondary hemorrhage is low, gynecologists do come across secondary hemorrhage of varying degrees of severity [1]. There are few studies which show the overall incidence of hemorrhage of $0.2-2 \%$ after hysterectomy which includes reactionary and secondary hemorrhage [1-4]. Our centre has been performing laparoscopic hysterectomies since 1994, and we encounter one or two cases of secondary hemorrhage per year in the second or third postoperative week which necessitates hospitalization and active treatment. We believe that this incidence is higher following laparoscopic hysterectomy than the other modes of hysterectomy. The purpose of this study is to estimate the cumulative incidence of secondary hemorrhage resulting from different modes of hysterectomy including abdominal, vaginal, and laparoscopic hysterectomies and to assess whether laparoscopic hysterectomy poses a greater risk. The second goal of this study is to describe the patient characteristics of those with secondary hemorrhage after hysterectomy and to identify the potential risk factors.

\section{Methods}

All women who underwent total laparoscopic hysterectomy (TLH) performed by the first author at Paul's Hospital 
between January 2004 and April 2012 and all cases of total abdominal hysterectomy (TAH) and vaginal hysterectomy (VH) performed by the second author from February 2010 to May 2012 at various private hospitals in Nanded were included in this study. The medical records of the patients were reviewed to ensure that those patients who had bleeding per vaginum between $24 \mathrm{~h}$ to 6 weeks after primary surgery and those requiring some intervention in the form of vaginal packing, vault suturing, laparoscopy, laparotomy, or embolization procedures were included in the analysis.

The institutional review board approved the data collection, aggregation, and analysis for this project. The following data were studied: age, parity, body mass index, indication for hysterectomy, size of uterus, details of surgical procedure, administration of antibiotics, time interval between hysterectomy and secondary hemorrhage, presenting symptoms, hemodynamic status, and type of intervention needed to manage the secondary hemorrhage.

Hysterectomies were categorized as TAH, VH, and TLH. All TLH were type IV E laparoscopic hysterectomies according to the classification system of the American Association of Gynecologic Laparoscopists [5]. TLH procedures were performed by dissecting the entire uterus laparoscopically; uterine arteries were coagulated with bipolar or Enseal (Ethicon Endosurgery, Inc.), and laparoscopic colpotomy was done with monopolar hook at a level above the uterosacral attachment, preserving the uterosacral arch. Vaginal cuff was closed vaginally in a continuous nonlocking fashion with Polysorb, Vicryl, or Dexon 1-0 size. Total abdominal hysterectomies were performed by division and ligation of pedicles with Vicryl 1-0. Uterosacral and cardinal ligament were divided and ligated with the same suture. Vault was sutured transversely with continuous or interrupted sutures while suspending the vault to the uterosacral and cardinal ligament at the angles. Vaginal hysterectomy was performed by clamping, dividing, and ligating the pedicles with Vicryl 1-0; the vault was sutured horizontally with continuous or interrupted sutures while suspending the vault to the uterosacral and cardinal ligament at the angles. All TLH patients received two doses of antibiotics, the first dose intraoperatively and the second dose postoperatively (fluoroquinolones and third generation cephalosporins). They were discharged on postoperative day1. All patients in TAH and $\mathrm{VH}$ group received antibiotics preoperatively and continued for 5 days. All TAH patients were discharged on day 7 after hysterectomy, and VH patients were discharged on day 4 which is a hospital protocol. Statistical analyses were performed using chi-square test, Kruskal-Wallis test, and ANOVA. A $p$ value of $<0.05$ was considered statistically significant.

\section{Findings}

A total of 3,757 hysterectomies were performed via all surgical modalities. One thousand six hundred twentythree had TLH, 1171 had undergone VH, and 963 had $\mathrm{TAH}$. Of the total 37 hemorrhages following hysterectomies, 23 were after TLH, 8 following VH, and 6 were after $\mathrm{TAH}$. The cumulative incidence of secondary hemorrhage due to different modes of hysterectomies were also analyzed (Table 1). The overall cumulative incidence of secondary hemorrhage after any type of total hysterectomies was $0.98 \%$. TLH was associated with the highest risk of secondary hemorrhage $(1.51 \%)$ followed by VH $(0.68 \%)$ and TAH group $(0.62 \%)$. The relative risk of secondary hemorrhage following TLH compared to TAH and VH were 2.3 and 2.1 , respectively. Both were statistically significant with $95 \%$ CI of 0.93 to 5.6 and 0.93 to 4.6. Patient characteristics and clinical presentation were also analyzed (Table 2).

The indications for the hysterectomies are depicted in Table 3 with the main indication being myoma uterus $(n=$ 18). Surgical details of patients are described in Table 4 . The average size of the uterus in the TLH group was $516.7 \mathrm{~g}$, and in the TAH and VH group, it was 140 and $142.5 \mathrm{~g}$, respectively, which was statistically significant. In patients with hemorrhage after TLH, bipolar was used in 12 cases, and in the remaining 11 patients, Enseal was used to coagulate the uterine pedicle. The median time interval between hysterectomy and secondary hemorrhage was 11 days in the TAH and $\mathrm{VH}$ groups and 13 days in the TLH group.

All patients presented with bleeding per vaginum of varying degrees. Two patients in TAH and VH group had pain abdomen along with bleeding. Three patients in TLH group and three patients in TAH group were in a state of hypovolemic shock at the time of hospitalization. Blood transfusions were needed in five patients in the TLH group and six patients each in the TLH and VH groups (Table 5).

Vaginal packing was sufficient to control bleeding in 14 patients in the TLH group, whereas seven patients required

Table 1 Types of hysterectomy and incidence of secondary hemorrhage

\begin{tabular}{llcl}
\hline $\begin{array}{l}\text { Type of } \\
\text { hysterectomy }\end{array}$ & $\begin{array}{l}\text { No. of secondary } \\
\text { hemorrhage }\end{array}$ & $\begin{array}{l}\text { Total no. of } \\
\text { hysterectomy }\end{array}$ & $\begin{array}{l}\text { Incidence of } \\
\text { hemorrhage in \% } \\
(95 \% \mathrm{CI})\end{array}$ \\
\hline $\mathrm{TLH}^{*}$ & 23 & 1,623 & $1.51(1.01-2.26)$ \\
$\mathrm{TAH}$ & 06 & 963 & $0.62(0.25-1.3)$ \\
VH & 08 & 1,171 & $0.68(0.32-1.3)$ \\
Total & 37 & 3,757 & $0.98(0.70-1.34)$ \\
\hline
\end{tabular}

$* p<0.0001($ TLH vs TAH $+\mathrm{VH})$ 
Table 2 Demography of patients with secondary hemorrhage after hysterectomy

\begin{tabular}{lllll}
\hline Variables & TLH (23) & TAH (06) & VH (08) & Total (37) \\
\hline Age & & & & \\
$\quad$ Mean \pm SD & $45.83 \pm 4.5$ & $48.83 \pm 7.1$ & $48.37 \pm 8.5$ & $46.9 \pm 5.9$ \\
Parity & & & & \\
$\quad$ Median (range) & $2(1-4)$ & $3.5(2-4)$ & $4(3-5)$ & $3.5(3-5)$ \\
1 & 04 & 0 & 0 & 04 \\
$\geq 2$ & 19 & 06 & 08 & 33 \\
Deliveries & & & & \\
$\quad$ Vaginal & 17 & 05 & 08 & 30 \\
$\quad$ Cesarean & 06 & 01 & 00 & 07 \\
BMI & & & & \\
$\quad$ Mean \pm SD & $27.22 \pm 3.7$ & $23.1 \pm 4.5$ & $26.5 \pm 4.8$ & $26.4 \pm 4.2$ \\
\hline
\end{tabular}

vault suturing. Laparoscopic coagulation of the uterine artery was done in one patient where the source of bleeding could not be identified vaginally. Uterine artery embolization was done twice in one patient to control the bleeding. Three patients each in the VH and TAH group were managed with vaginal packing alone. Four patients in the VH group had vault hematoma which was diagnosed on ultrasound and drained vaginally. In the TAH group, three patients were subjected to laparotomy. Out of the three patients, one had rectus sheath hematoma which had tracked down into the vagina and in the other two patients as the source of bleeding could not be identified; all pedicles were religated. On further evaluation, one of these patients was diagnosed to have chronic idiopathic thrombocytopenic purpura (Table 5).

Resumption of sexual intercourse was not found in any of these patients as all of them presented within 6 weeks after surgery. The period of abstinence advised was 68 weeks after TLH and 12 weeks after TAH and VH. Early resumption of regular activities was observed in TLH and VH patients. TAH patients resumed regular activities only after 45 days.

Table 3 Indications for hysterectomy

\begin{tabular}{lrrrr}
\hline Indication for hysterectomy & TLH & TAH & VH & Total \\
\hline Myoma & 16 & 1 & 1 & 18 \\
Cervical pathology & 0 & 2 & 1 & 3 \\
Myoma with endometriosis & 2 & 0 & 0 & 2 \\
Myoma with ovarian pathology & 1 & 0 & 0 & 1 \\
Adenomyosis & 1 & 0 & 1 & 2 \\
DUB & 1 & 1 & 1 & 3 \\
Ovarian pathology & 0 & 1 & 0 & 1 \\
Post menopausal bleeding & 1 & 1 & 0 & 2 \\
Prolapse uterus & 1 & 0 & 4 & 5 \\
Total & 23 & 06 & 08 & 37 \\
\hline
\end{tabular}

\section{Discussion}

Hemorrhage after hysterectomy is a life-threatening complication [1]. There are few published reports on the incidence of secondary hemorrhage after hysterectomy. In our study of 3,757 hysterectomies, 37 patients $(0.98 \%)$ had secondary hemorrhage. TLH had a higher incidence of $1.51 \%$, whereas VH $(0.68 \%)$ and TAH $(0.62 \%)$ had a lower incidence.

Donnez et al. in his series of 2,596 laparoscopic hysterectomies including laparoscopic subtotal hysterectomy and TLH had a lower incidence $(0.1 \%)$ of hemorrhage [6]. Wattiez et al. in a retrospective comparative study on the effect of learning curve on the outcome of laparoscopic hysterectomy done during 1989-1995 and 1996-1999 on 695 and 952 women, respectively, concluded that there was a substantial decrease in the major complication rates from 5.6 to $1.3 \%$, excessive hemorrhage from 1.9 to $0.1 \%$ ( $p<$ $0.005)$, respectively [7]. Both studies did not specify the type of hemorrhage. Nezhat et al. in his comparative study of laparoscopic-assisted vaginal hysterectomy and abdominal hysterectomy of 20 patients showed that LAVH group had shorter duration of hospitalization ( 2.4 vs 4.4 days ), more rapid recuperation ( 3 vs 5 weeks ) and fewer complications [8]. Canis et al. in a retrospective cohort study on 680 patients found that seven cases (1.03\%) had postoperative hemorrhage following hysterectomy, two cases involved vaginal laceration and five intraperitoneal hemorrhages [9].

Holub et al. reported two cases $(0.17 \%)$ of secondary hemorrhage in his series of 1,167 patients with laparoscopic hysterectomy and vaginal hysterectomy [4]. Wilke et al. reported an incidence of $0.23 \%$ of secondary hemorrhage following $\mathrm{VH}$ and laparoscopic hysterectomy [1]. In an earlier study by Bhattacharya et al., the incidence of secondary hemorrhage was $0.45 \%$ after vaginal hysterectomies [3]. In our study, the incidence of secondary hemorrhage after TAH and $\mathrm{VH}$ is comparable but is higher in the TLH group. Infrequent occurrence of secondary hemorrhage, failure to report to the centre where hysterectomy was performed or nondocumentation of cases may be the possible reasons for lower incidence reported in the literature.

Possible factors which may play a role in secondary hemorrhage are patient characteristics, size of the uterus, surgical techniques, vaginal vault infection, and early resumption of physical activity.

Age and BMI of patients in the three groups were not statistically significant and thus unlikely to contribute to the increased incidence of secondary hemorrhage in the TLH group. In the present study, the size of the uterus was significantly higher among the TLH group. Out of the 18 cases of secondary hemorrhage in the TLH group, 16 had 
Table 4 Surgical details

\begin{tabular}{|c|c|c|c|c|}
\hline & TLH (23) & TAH (06) & VH (08) & Total (37) \\
\hline \multicolumn{5}{|l|}{ Previous surgeries } \\
\hline No & 13 & 01 & 04 & 18 \\
\hline Yes & 10 & 05 & 04 & 19 \\
\hline Total & 23 & 06 & 08 & 37 \\
\hline \multicolumn{5}{|l|}{ Size of uterus* } \\
\hline Mean weight \pm SD & $516.7 \pm 443.1$ & $140 \pm 47.32$ & $142.5 \pm 66.9$ & $374.3 \pm 394.2$ \\
\hline \multicolumn{5}{|l|}{ Energy source of uterine artery } \\
\hline Bipolar & 11 & 0 & 0 & 11 \\
\hline Enseal & 12 & 0 & 0 & 12 \\
\hline Suturing & 0 & 06 & 08 & 14 \\
\hline Total & 23 & 06 & 08 & 37 \\
\hline \multicolumn{5}{|l|}{ Energy source for vault } \\
\hline Monopolar & 23 & 0 & 0 & 23 \\
\hline None & 00 & 06 & 08 & 14 \\
\hline Total & 23 & 06 & 08 & 37 \\
\hline \multicolumn{5}{|l|}{ Post-op complications } \\
\hline Fever & 2 & 0 & 2 & 2 \\
\hline Post-op blood transfusions & 0 & 2 & 3 & 5 \\
\hline Reactionary hemorrhage & 0 & 0 & 1 & 1 \\
\hline Urinary retention & 0 & 0 & 2 & 2 \\
\hline Cough/constipation & 1 & 0 & 1 & 1 \\
\hline Pain abdomen & 0 & 2 & 2 & 4 \\
\hline
\end{tabular}

${ }^{*} p$ value $<0.02$

fibroid uterus with mean weight of $516 \pm 443$. Though TLH is a feasible and safe technique in cases of enlarged uteri, which permits avoidance of laparotomy with evident benefits for the patients [10], high vascularity and large-sized vessels may be responsible for the increased incidence of secondary hemorrhage [11].

Table 5 Clinical presentation and treatment of secondary hemorrhage

TLH (23) TAH (6) VH (8) Total (37)

\begin{tabular}{lrrrr}
\hline Severity of hemorrhage & & & & \\
$\quad$ Mild $\leq 200 \mathrm{ml}$ & 11 & 1 & 3 & 15 \\
$\quad$ Profuse $>200 \mathrm{ml}$ & 12 & 5 & 5 & 27 \\
Hemodynamic status & & & & \\
$\quad$ Stable & 20 & 3 & 8 & 34 \\
$\quad$ Hypovolemic shock & 3 & 3 & 0 & 3 \\
Treatment & & & & \\
Blood Transfusion & 5 & 6 & 6 & 17 \\
$\quad$ Vaginal packing & 14 & 3 & 3 & 20 \\
$\quad$ Vault suturing & 7 & 0 & 1 & 7 \\
$\quad$ Laparoscopy & 1 & 0 & 0 & 1 \\
$\quad$ Laparotomy & 0 & 3 & 0 & 3 \\
$\quad$ Uterine artery embolization & 1 & 0 & 0 & 1 \\
$\quad$ Vault hematoma drainage & 0 & 0 & 4 & 4 \\
\hline
\end{tabular}

The time interval between hysterectomy and onset of secondary hemorrhage ranged between 3 and 22 days. The median interval for the TLH group was 13 days, and for the TAH and $\mathrm{VH}$ groups, it was 11 days. Of the few reported cases in literature, the time interval varied from 3 to 18 days $[1,2,4]$.

As the majority of patients could be managed with vaginal packing alone, it implies that the source of bleeding could be from the vaginal cuff. Any obvious vault bleeder can be secured with suture. Laparoscopic surgery in postoperative bleeding after hysterectomy is feasible and may be recommended if the source of bleeding cannot be identified by vaginal examination or if the symptoms indicate that the source of bleeding is intraabdominal [4]. Laparoscopy provides good magnification which allows closer inspection and a more precise use of bipolar coagulation or suturing for management of hemorrhage [1]. In our study, one patient underwent laparoscopic coagulation of uterine artery in the TLH group. Laparotomy may be done where laparoscopic surgical skills are not available [1]. Emergency therapeutic arterial embolization is a safe and effective minimal invasive procedure for patients developing postoperative hemorrhage after gynecological laparoscopic surgery [12]. In our study, one patient with secondary hemorrhage in the TLH group underwent uterine artery embolization twice. Vaginal vault dehiscence is one of the rare complications after hysterectomy [13]. In our study, we did not come across any vaginal vault dehiscence. 
The source of bleeding in secondary hemorrhage can be from uterine vessels or descending cervical/vaginal vessels. The source of bleeding was a uterine vessel in two out of 23 cases in the TLH group and two patients in the TAH group. Use of energy source for the uterine vessels is unlikely to increase the incidence of secondary hemorrhage after TLH. Occasionally, uterine artery pseudoaneurysm can cause delayed heavy vaginal bleeding after laparoscopic hysterectomy [14]. Monopolar energy is used for colpotomy in all patients in the TLH group. The use of thermal energy may result in increased tissue damage to the vaginal cuff $[2,15]$. Vault bleeding was responsible for secondary hemorrhage in 21 out of 23 cases. Hence, the usage of energy source for the vault may be responsible for higher incidence of secondary hemorrhage after TLH. It may be advisable to minimize the use of thermal energy so that the tissue is not over desiccated [2].

The exact cause for increased incidence of secondary hemorrhage after TLH is unknown. We may hypothesize that the application of thermal energy to tissues may cause more tissue necrosis and devascularization than sharp colpotomies in the TAH and VH groups. Treatment of secondary hemorrhage via vaginal approach in the form of packing, suturing, or drainage of hematoma appears to be feasible as initial intervention to control bleeding. Also, care must be taken while performing TLH in large uteri and limiting the over enthusiastic use of thermal energy. TLH is still recommended over abdominal hysterectomy because of obvious advantages [13, 16, 17].

Limitations of the present study are that it is a retrospective observational study performed in two different places, in two different time periods, and by two different surgeons. Since the incidence of secondary hemorrhage is very low, longer duration was included for the TLH series. So the patient groups were not directly comparable. When the study was started, the first author had 10 years of experience in doing laparoscopic hysterectomy; it is unlikely that the learning curve has affected the outcome. Further prospective randomized control trial studies are needed to validate our results.

\section{Conclusion}

Our data suggests that secondary hemorrhage is rare but may occur more often after TLH than after other hysterectomy approaches. Large size of uteri, excessive use of energy source for uterine artery, and colpotomy may play a role. The comparison of different energy sources like ultrasonic (harmonic) or bipolar with scissors for colpotomy in TLH is worth studying. TLH is a feasible and safe technique in cases of enlarged uteri, which permits avoidance of laparotomy with evident benefits for the patients [10].
Conflict of interest On behalf of all the authors, the corresponding author states that there is no conflict of interest. The authors alone are responsible for the content and writing of the paper.

Author's statement of responsibility All the authors of this study were actively involved in the design of the study, data collection and analysis, statistical analysis, and manuscript preparation.

Informed consent Informed consent was obtained from all patients for being included in the study.

\section{References}

1. Wilke I, Merker A, Schneider A (2001) Laparoscopic treatment of hemorrhage after vaginal hysterectomy or laparoscopically assisted vaginal hysterectomy (LAVH). Surg Endosc 15:11441146

2. Miranda CS, Carvajal AR (2003) Complications of operative gynecological laparoscopy. JSLS 7:53-58

3. Bhattacharya MS, Shinde SD, Narwekar MR (1978) Complications of vaginal hysterectomy (analysis of 1105 cases). J Postgrad Med 24:221-225

4. Holub Z, Jabor A (2004) Laparoscopic management of bleeding after laparoscopic or vaginal hysterectomy. JSLS 8:235-238

5. Olive DL, Parker WH, Cooper JM, Levine RL (2000) The AAGL classification system for laparoscopic hysterectomy. Classification committee of the American association of gynecologic laparoscopists. J Am Assoc Gynecol Laparosc 7:9-15

6. Donnez J, Jadoul P, Donnez O, Squifflet J (2007) What is the preferred route for hysterectomy?-proposition: most uteri can be removed by laparoscopy! In proceedings of the 9th world congress on controversies in obstetrics. Gynecology and Infertility, Barcelona, Spain

7. Wattiez A, Soriano D, Cohen SB, Nervo P, Canis M, Botchorishvilli $R$ et al (2002) The learning curve of total laparoscopic hysterectomy; comparative analysis of 1647 cases. J Am Assoc Gynecol Laparosc 9:339-345

8. Nezhat F, Nezhat C, Gordon S, Wilkins E (1992) Laparoscopic versus abdominal hysterectomy. J Reprod Med 37(3):247-250

9. Canis M, Botchorishvili R, Ang C, Rabischong B, Jardon K, Wattiez A, Mage G (2008) When is laparotomy needed in hysterectomy for benign uterine disease? J Minim Invasive Gynecol 15(1):38-43

10. Fiaccavento A, Landi S, Barbieri F, Zaccoletti R, Tricolore C, Ceccaroni M (2007) Total laparoscopic hysterectomy in cases of large uteri: a retrospective comparative study. J Minim Invasive Gynecol 14:559-563

11. Bonilla DJ, Manis L, Whitaker R, Crawford B, Finan M, Magnus M (2007) Uterine weight as a predictor of morbidity after a benign abdominal and total laparoscopic hysterectomy. J Reprod Med 52(6):490-498

12. Takeda A, Koyama K, Mori M, Sakai K, Mitsui T, Nakamura H (2008) Diagnostic computed tomographic angiography and therapeutic emergency transcatheter arterial embolization for management of postoperative hemorrhage after gynecologic laparoscopic surgery. J Minim Invasive Gynecol 15(3):332341

13. Hur HC, Guido RS, Mansuria SM, Hacker MR, Sanfilippo JS, Lee TT (2007) Incidence and patient characteristics of vaginal cuff dehiscence after different modes of hysterectomies. J Minim Invasive Gynecol 14:311-317

14. Miligkos DS, Louden K, Page A, Behrens R (2013) Uterine artery pseudoaneurysm following laparoscopic hysterectomy. An 
unusual cause of delayed heavy vaginal bleeding. Gynecol Surg. doi:10.1007/s10397-031-0785-5

15. Memon MA (1994) Surgical diathermy. Br J Hos Med 52:403-408

16. Wiser A, Holcroft CA, Tulandi T, Abenhaim HA (2013) Abdominal verses laparoscopic hysterectomies for benign disease: evaluation of morbidity and mortality among 465,798 cases. Gynecol Surg. doi:10.1005/s10397-078-9

17. Karaman YC, Bingol B, Günenç Z (2007) Prevention of complications in laparoscopic hysterectomy: experience with 1120 cases performed by a single surgeon. J Minim Invasive Gynecol 14:78-84 